\title{
A Remote Monitoring and Decision-Making System for Vehicle Rental
}

\author{
De Long, Jian-Jun Yi, Fei-xiang Xu, Xiao-Ming Zhu \\ Department of Mechanical Engineering, East China University of Science and Technology, ECUST, Shanghai, China; \\ E-mail: delongecust@foxmail.com; jjyi@ecust.edu.cn; xufeixiangsdut@163.com; zxmin4236@163.com
}

\begin{abstract}
Vehicle rental service is being developed greatly in recent 10 years. A remote monitoring and decision-making system is proposed in this paper. This system can help the lessors to supervise their vehicles in real time. It also facilitates the customers to acquire the real time status of the rented vehicle, such as the working conditions, locations of the vehicle, costs, etc. The system consists of a data acquisition terminal and a remote monitoring and decision-making system. The data acquisition terminal is responsible for collecting and transmitting the vehicle fault information from the OBD (OnBoard Diagnostics) interface and the vehicle location information from GPS module. And the remote monitoring and decision-making system integrated a fault expert system based on ontology with GIS platform, thus remote monitoring and diagnosis for vehicle can be achieved, which can provide effective suggestions when vehicle failure occurs. Ontology engineering theory is introduced in order to improve the intelligence and scalability of the expert system for fault diagnosis. Finally, some vehicle rental company is taken as an example to demonstrate the system functions. The result is proved that this system presents many advantages such as effective remote monitoring and decision making, and accurate ontology-based analysis.
\end{abstract}

Keywords- fault diagnosis, ontology, wireless communication, monitoring and decision making

\section{INTRODUCTION}

With the development of vehicle rental service, the realtime vehicle running status monitoring is required urgently. On one hand, it is a great potential safety hazard for drivers if they can't know the vehicle's running status, they cannot maintenance and repair the vehicle in time on the other hand it is important to help the lessor company to improve the supervision of the vehicles.

OBD means an on-board diagnostic system for fault diagnosis which has the capability of identifying the possible reasons by analyzing fault codes stored in computer memory and analyzing the information from the vehicle CAN bus [1]. GPS (Global Positioning System) integrated with GIS (Geographic Information System) can display the location of the vehicle on the map [2].

Ontology is derived from the philosophy. In recent decades, it is widely used in artificial intelligence, computer language and database theory with the development of information technology. It can provide common, domain knowledge that can be shared and provide support for further derivation. It has rich semantic knowledge, formalized expression which can realize the reuse and sharing of knowledge, and it could also provide support for further derivation [3].

Based on this, a remote monitoring and decision making system is proposed in this paper. This system can realize functions including remote monitoring and diagnosis, it provide effective suggestions when vehicle failure occurs, and it is provided to users in the form of App and website. It does not only makes the vehicle supervision easier for the vehicle lessor company but also improves the convenience of car maintenance and reduces maintenance costs greatly. So this system has a very broad application prospects.

\section{OVERALL ARCHITECTURE}

The overall architecture of the system is shown in Figure 1 . The system mainly consists of a data acquisition terminal and a remote monitoring and decision making system. The data acquisition terminal is installed on the car, and vehicle fault information gathered by OBD interface combined with vehicle location information gathered by GPS module formed data packets, then the data is sent to the remote server through the GPRS communication module. The data packets will be parsed and stored in the database on the remote server, the remote monitoring and decision making system will complete fault diagnosis by fault diagnosis expert system based on ontology according to the fault information such as fault code. GIS monitoring system will display the location and diagnosis result. Remote terminal such as computers and mobile phones can have access to the system through the network. The interface of the GIS monitoring system will pop up an alarm which shows the fault code, fault phenomenon, fault source, fault reason, maintaining method and the best maintenance station. This information will be pushed to the mobile phone at the same time.

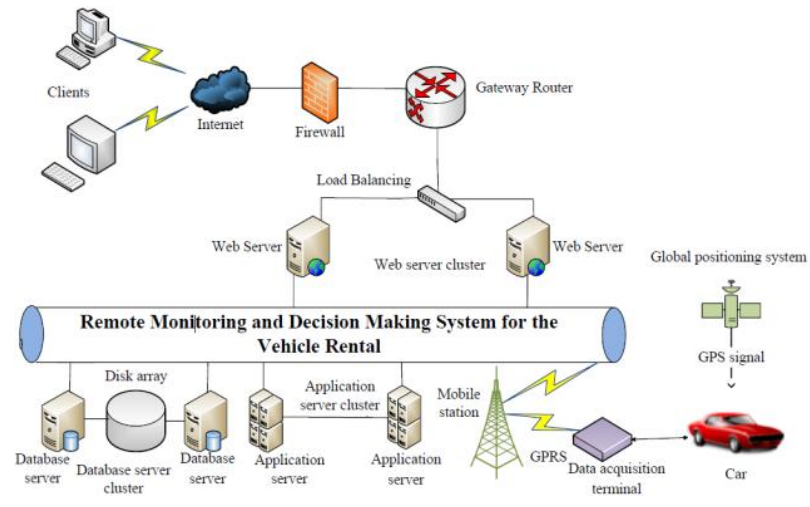

Figure 1. The overall framework of the proposed system. 


\section{IMPLEMENTATION OF REMOTE MONITORING AND DECISION MAKING SYSTEM}

\section{A. Network Communication and Data Processing}

\section{1) Network communication}

The data acquisition terminal gathered the fault information and location information through the GPRS communication module and then sends the data to the remote server. The remote server completed data receiving by Socket. As there are a large quantity of data acquisition terminal nodes, this system used UDP (User Datagram Protocol) transport protocols [4].

\section{2) Database design}

Figure 2 shows the architecture of the remote monitoring center database that contains the user information module, position information module, and fault diagnosis module according to the system requirements. User information module contains user roles, user basic information, and user operating authority information. Location information module mainly contains the information obtained from the GPS module, such as longitude, latitude, etc. Fault diagnosis information module mainly contains the basic information of the vehicle such as the vehicle manufacturer, model and engine information, standard OBD fault code retrieval table, and the fault information gathered by OBD interface.

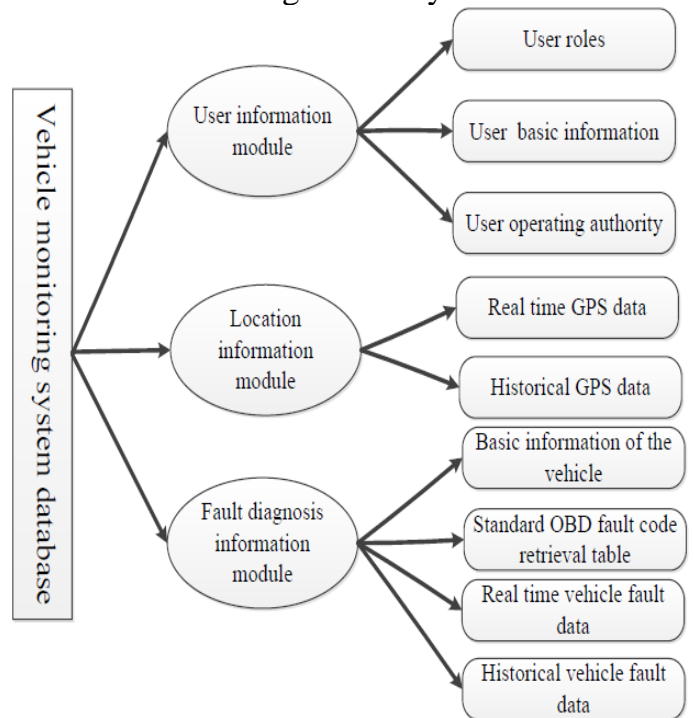

Figure 2. The architecture of the remote monitoring center database.

\section{3) Data transmission}

The upload data format from the data acquisition terminal is shown in table I:

TABLE I. DATA FORMAT OF THE UPLOAD DATA

\begin{tabular}{|c|c|c|c|c|c|c|c|}
\hline $\begin{array}{c}\text { Start } \\
\text { bit }\end{array}$ & $\begin{array}{c}\text { Signal } \\
\text { ing }\end{array}$ & & & $I D$ & $\begin{array}{r}\text { OBD } \\
\text { data }\end{array}$ & Check & End bit \\
\hline $29 \quad 29$ & F1 & 00 & 28 & & (34Byte & & OD \\
\hline
\end{tabular}

The useful information is the data from the OBD. The information contained in the packet has time, latitude, longitude, speed, direction Angle, fault code and so on. The data format is shown in table II:
TABLE II. DATA FORMAT OF THE OBD DATA

\begin{tabular}{|l|c|c|c|c|c|c|}
\hline Time & Latitude & Longitude & Speed & $\begin{array}{c}\text { Direction } \\
\text { angle }\end{array}$ & $\begin{array}{c}\text { Fault } \\
\text { information } \\
\text { (variable) }\end{array}$ & $\begin{array}{c}\text { Reserved } \\
\text { bits }\end{array}$ \\
\hline 6Byte & 4 Byte & 4 Byte & $2 \mathrm{Byte}$ & $2 \mathrm{Byte}$ & & \\
\hline
\end{tabular}

Remote server parses the data from the received data packet based on the above communication protocol.

\section{B. GIS Monitoring System}

GIS monitoring system will display the running status of the vehicle which includes the operating conditions and locations. The system is mainly divided into the following three modules.

\section{1) User management}

This system has three user permissions include drivers, supervision departments, and experts. The drivers can know the real-time running status of the vehicle, and get effective suggestions when vehicle failure occurs such as the best maintenance station and other maintenance advices. The supervision departments can monitor the running status of all the vehicles and search the history fault information of all the vehicles for management and decision-making. Experts can add more fault diagnosis knowledge into the knowledge bases. Using the system in respective permissions can greatly improve the security of the system.

2) Position monitoring

This monitoring system can monitor the real time vehicle's position and velocity and search historical track. The lessors can monitor the position of the car to confirm whether it is beyond the allowed area.

\section{3) Operating condition monitoring}

When the vehicle failure occurs, the monitoring system will show vehicle fault information in time, including fault code, fault source, fault reason, fault phenomenon and repair recommendation. All of this information is from the fault diagnosis expert system based on ontology, and this system will be introduced in the next chapter.

\section{Fault Diagnosis Expert System Based on Ontology}

Applying ontology to vehicle remote fault diagnosis expert system, can make automobile fault diagnosis simple and effective, and give reasonable maintenance advice when vehicle failure occurs.

1) The overall framework of the fault diagnosis expert system based on ontology

Figure 3 shows the overall framework of the fault diagnosis expert system based on ontology. The system includes knowledge acquisition layer, diagnosis layer and decision support layer. The knowledge acquisition layer gets the basic data for building the ontology model, including the fault codes stored in computer memory and the information from the vehicle CAN bus. The diagnosis layer is mainly responsible for diagnosis reasoning based on ontology, it will get the reasoning results by the knowledge acquisition layer in combination with certain reasoning mechanism. According to the result of diagnosis layer, the decision support layer mainly gives the corresponding repair recommendation, and the result will be sent to the user. 
Finally, the conclusions will be stored and updated the knowledge base.

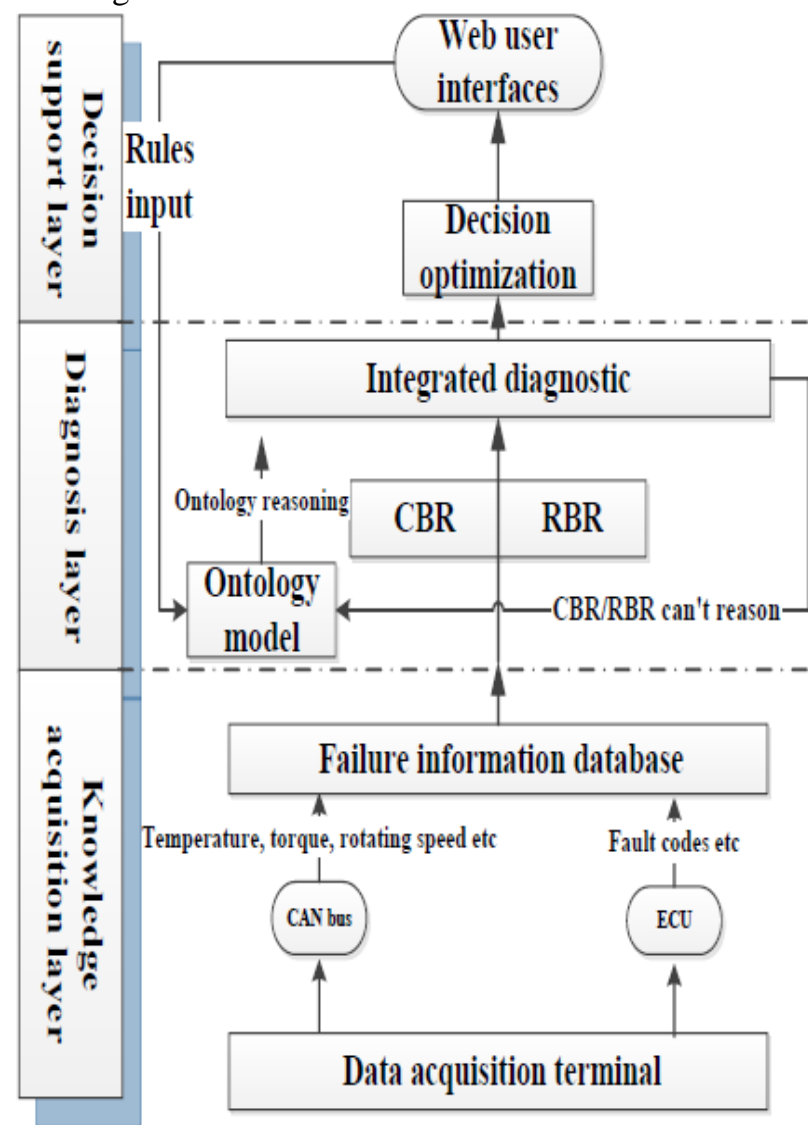

Figure 3. The overall framework of the fault diagnosis expert system based on ontology.

2) The establishment of the vehicle failure ontology model

This paper adopts Seven-Step Method to build the vehicle failure domain ontology model. Seven-Step Method, developed by the Medical Information Center of Stanford University, is a relatively mature method to build domain ontology [5]. Figure 4 shows the ontology model of the vehicle failure. It mainly consists of fault code class, fault phenomenon class, fault source class, fault reason class, and maintaining method class. The fault phenomenon class consists of the subclasses of cannot start, start difficultly, dysfunctional in idle speed, accelerate badly, engine stall and other fault phenomenon. The fault source class consists of the subclasses of intake air control system, fuel supply system, injector, electronic control system, idle control system, and other fault sources [6]. The fault reason class consists of the subclasses of start system fault, ignition system fault, fuel injection fault, intake system fault, and other fault reasons [7]. The maintaining method class consists of the subclasses of sparkplug inspection, cylinder cap inspection, intake tube inspection, and other inspections. This paper uses Pellet 1.5.2 to test the validity of ontology organizational structure and class property definition after building the ontology model, and no abnormal situation occurred in the testing process. Therefore, the ontology model is correct and reasonable.

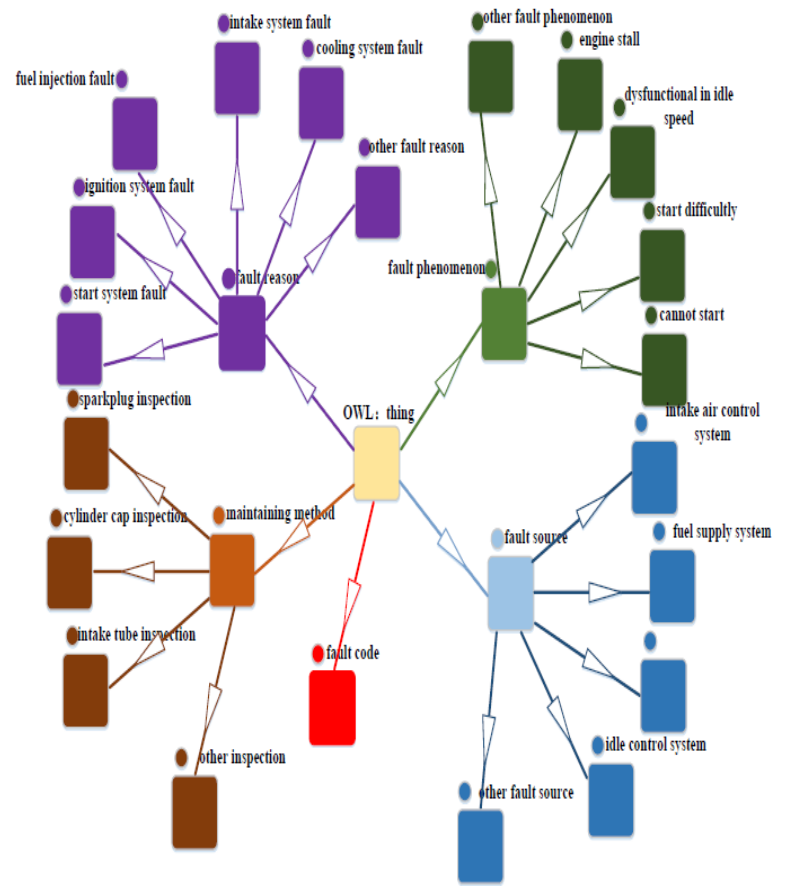

Figure 4. Semantic processing vehicle failure information ontology model.

3) Implementation of vehicle failure ontology analysis

a) Analysis of vehicle failure ontology based on jena

This program uses createOntologyModel() method for ontology instantiation and opens the .owl document by file stream. The program code is as follows:

OntModel model

ModelFactory.createOntologyModel();

Private static String filenameinput =

"CarDiagnosis.owl";

InputStream streaminput =

FileManager.get().open(filenameinput);

model.read(streaminput, "");

So far, the reading and parsing of the ontology of vehicle failure is completed.

b) Implementation of query inference based on sparql and jena

Sparql(Simple Protocol and RDF Query Language) is a query language service for $\mathrm{RDF}$, and it can get the information existing in model without inferring function [8]. This paper chooses the query method of SELECT - FORM - WHERE to query the vehicle fault code table. The program code is as follows:

string url = "http://CarDiagnosis.owl";

string[] str3=\{ "DiagnosisCode_I" $\}$;

queryString = "Select ? Fault_Code1 " + "WHERE " $+"\{<"+\operatorname{str}+" \# "+\operatorname{str} 3[0]+"><"+\operatorname{str}+$

"\#Has_Value_Code>? Fault_Code1. \}"

It will get the fault reason by Sparql querying that compared fault code input with car fault code standard table, but it do not infer the relationship of fault codes with 
maintenance method, and the fault phenomena with fault code. Therefore, some rules should be established at Jena reasoning machine [9].

Rule1: (? x Originate ? y)(? z Repairing ? y) $\longrightarrow($ ? z Repairing ? $\mathrm{x})$

Rule2: (? x Originate ? y)(? z Repairing ? y) (? m Solved by ? z) $\longrightarrow$ (? x Stand for ? m)

(? $\mathrm{x}$ Originate ? y) means fault code $\mathrm{x}$ originated from fault source $\mathrm{y},($ ? z Repairing ? y) means the maintenance method $\mathrm{z}$ for repairing the fault source $\mathrm{y},($ ? $\mathrm{m}$ Solved by ? $\mathrm{z})$ means fault phenomenon $\mathrm{m}$ can be solved by the maintenance method z. (? x stand for ? z) means fault code x stand for the fault phenomenon $\mathrm{m}$.

\section{SYSTEM VALIDATION}

This research takes some vehicle of the rental company as an example to implement the system validation. The results show that the remote monitoring center can receive the data from data acquisition terminal effectively, and the GIS monitoring system can monitor the real time running status of the vehicle which includes the operating conditions and locations. The system can provide effective suggestions when vehicle failure occurs. As shown in Figure 5, the database shows the real-time data of the vehicle received from the data acquisition terminal. As shown in Figure 6, the monitor map shows the real time vehicle's position, the fault code P0174, and the diagnosis that filter blocked, the phenomenon of engine powerless may happen, recommend replacing filter. The engine powerless situation did exist in the actual operation process, and problem solved after the replacement of filter. Besides, the system provided a suggestion of the best maintenance station according to the vehicle models and manufacturers. In addition, the system provided the function of querying the history information including the historical track and the historical fault for the convenience of company operation management. Figure 7 shows the fault code, the fault reason, fault phenomenon and maintenance method of all vehicles has occurred within a certain time. Finally, the fault information and the maintenance advice will be pushed to the mobile phone. Figure 8 shows the pushed message in detail.

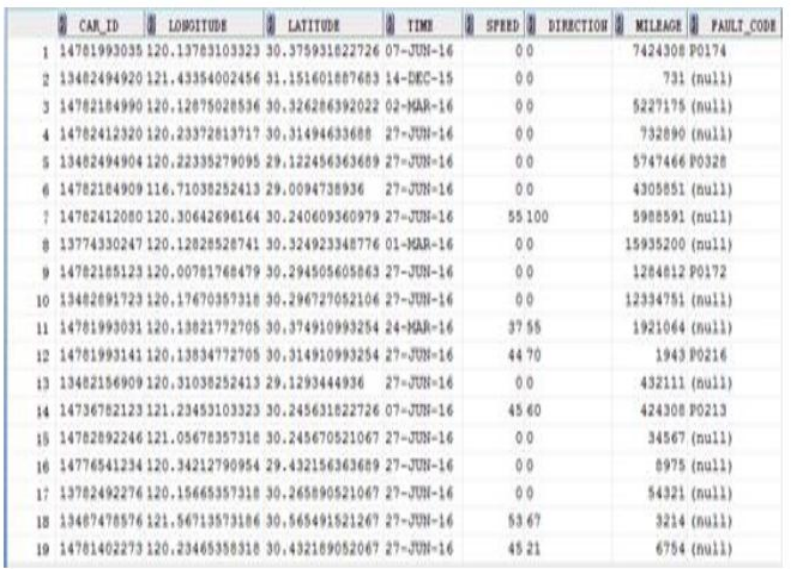

Figure 5. The real-time data of all vehicle.

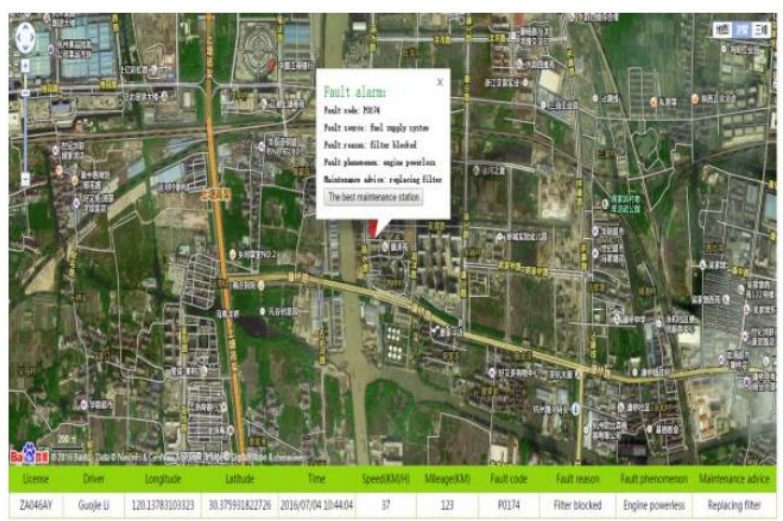

Figure 6. Interfaces of PC application: the monitoring of vehicle's running status.

\begin{tabular}{|c|c|c|c|c|c|}
\hline lagenterte & ithin & that & it & ithera & Whatita \\
\hline 遍 & mathin & III & thesatid & Ifye日e. & $\begin{array}{l}\text { Whinged } \\
\text { if }\end{array}$ \\
\hline$y$ & Dahyn & 四 & Inxiditute & Lapiatia & 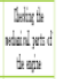 \\
\hline $2 y$ & Mahn & $\mathrm{n}$ & 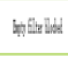 & What & 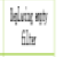 \\
\hline Da & Mahn & t8 & thintży & Whation & Heinpors \\
\hline we & ESCh14! & m & Derat & byeses & Whangix \\
\hline
\end{tabular}

Figure 7. Interfaces of $\mathrm{PC}$ application: the querying of history information of all vehicle.
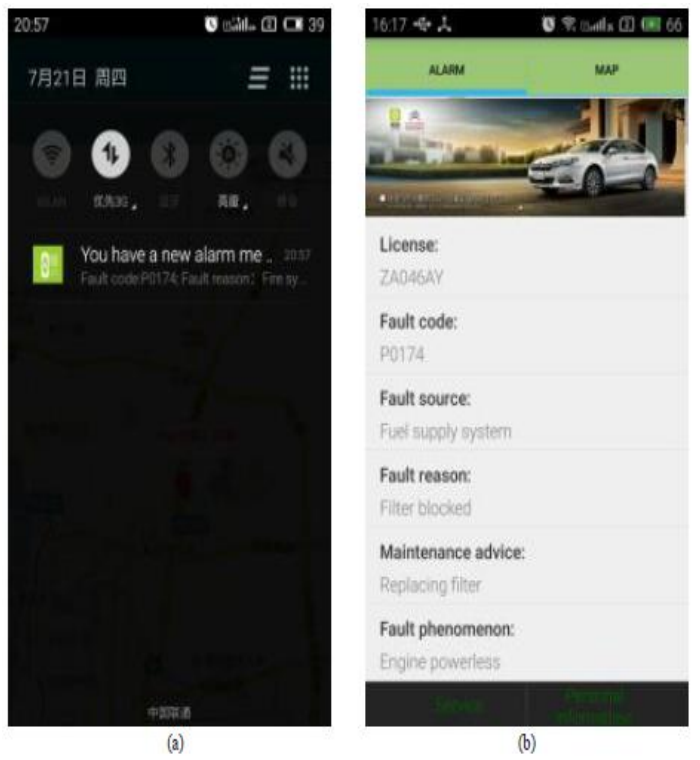

Figure 8. Interfaces of APP: the pushed message.

\section{CONCLUSIONS}

At present, there are few research on the car remote fault diagnosis reasoning based on ontology. Existing research can only monitor the location, not monitor the running state and make decision. We proposed a remote monitoring and 
decision making system for the vehicle rental based on ontology combines OBD, GPS, GPRS and GIS in this paper. The test results showed that the system had many advantages such as effective remote monitoring and decision making after parsing the received data. This system has higher application value in the management, security operation of the vehicle rental company.

\section{ACKNOWLEDGMENT}

This paper was supported by the Research Foundation of Science and Technology Commission of Shanghai under Grant No. 10DZ1500200, the Natural Science Fund of China (NSFC) under Grant Nos. 50975088, 51275173, 51575186 and 51210105018, the Fundamental Research Funds for the Central Universities under Grant No. WH0913009, Shanghai Pujiang Program under Grant No. PJ201000353, and Shanghai Software and IC industry Development Special Fund under Grant No. 120493.

\section{REFERENCES}

[1] Jianqiu Li. Automobile Electronics [M]. Beijing: Tsinghua University Press, 2006, pp. 327
[2] Yanhui Du. Design and Implementation of Vehicle Monitoring System Based on GIS [D]. Changchun: Jilin University, 2006.

[3] Xiulan Zhang, Ling Jiang. The concept review of ontology research [J]. Journal of the China Society for Scientific and Technical Information. vol. 26, no. 4, Aug. 2007, pp. 527-530, doi: 10.3969/j.issn.1000-0135.2007.04.008.

[4] Jianhua Li. The Guide of Visual C\# 2005 [M]. Beijing: Publishing House of Electronics Industry, 2008, pp. 288.

[5] F. N. Natalya and L. M. Deborah, "Ontology Development 101: A Guide to Creating Your First Ontology," 2000, http: // protege.stanford.edu /publications /ontology_development/ontology101.pdf.

[6] Lirong Wang. Study on Diagnosis Expert System of Electric Controlled Engine [D]. Qinhuangdao: Yanshan University, 2007.

[7] Lifang Liu.System of Fault Diagnosis of Automobile Engine [D]. Taiyuan:TaiYuan University of Technology,2012.

[8] S. Shekarpour, S. Auer, A.-C. N. Ngomo, D. Gerber, S. Hellmann, and C. Stadler, "Generating SPARQL queries using templates" Web Intelligence and Agent Systems, vol. 11, no. 3, pp. 283-295, 2013. View at Publisher - View at Google Scholar · View at Scopus.

[9] J. Wu and Y. Huang, "Reasoning with ontology model based on Jena" Intelligent Information Management, vol. 1, no. 1, pp. 24-29, 2009. View at Publisher · View at Google Scholar. 